\title{
Fulminant hepatitis and elevated levels of sIL-2R in thyroid storm
}

\section{Yuri Tanaka*, Taisuke Uchida*, Hideki Yamaguchi, Yohei Kudo, Tadato Yonekawa and Masamitsu Nakazato}

Division of Neurology, Respirology, Endocrinology and Metabolism, Department of Internal Medicine, Faculty of Medicine, University of Miyazaki, Miyazaki, Japan

*(Y Tanaka and T Uchida contributed equally to this work)

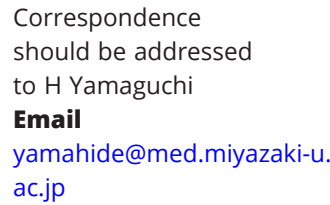

\section{Summary}

We report the case of a 48-year-old man with thyroid storm associated with fulminant hepatitis and elevated levels of soluble interleukin-2 receptor (sIL-2R). Fatigue, low-grade fever, shortness of breath, and weight loss developed over several months. The patient was admitted to the hospital because of tachycardia-induced heart failure and liver dysfunction. Graves' disease with heart failure was diagnosed. He was treated with methimazole, inorganic iodide, and a $\beta$-blocker. On the day after admission, he became unconscious with a high fever and was transferred to the intensive care unit. Cardiogenic shock with atrial flutter was treated with intra-aortic balloon pumping and cardioversion. Hyperthyroidism decreased over 10 days, but hepatic failure developed. He was diagnosed with thyroid storm accompanied by fulminant hepatitis. Laboratory investigations revealed elevated levels of sIL-2R ( $9770 \mathrm{U} / \mathrm{mL})$. The fulminant hepatitis was refractory to plasma exchange and plasma filtration with dialysis, and no donors for liver transplantation were available. He died of hemoperitoneum and gastrointestinal hemorrhage due to fulminant hepatitis 62 days after admission. Elevated circulating levels of sIL-2R might be a marker of poor prognosis in thyroid storm with fulminant hepatitis.

\section{Learning points:}

- The prognosis of thyroid storm when fulminant hepatitis occurs is poor.

- Liver transplantation is the preferred treatment for fulminant hepatitis induced by thyroid storm refractory to plasma exchange.

- Elevated levels of soluble interleukin-2 receptor might be a marker of poor prognosis in patients with thyroid storm.

\section{Background}

Thyroid storm is a rare, life-threatening complication of Graves' disease (1). Liver dysfunction in thyroid storm is induced by hepatic ischemia, dysfunction of hepatic metabolism, direct injury from thyrotoxicity, and antithyroid drugs (2). Fulminant hepatitis with thyroid storm causes multiple organ failure and is associated with poor prognosis.

Interleukin-2 (IL-2) is a cytokine produced by CD4positive T cells. IL-2 binds to IL-2 receptor expressed on the cell surface, thereby activating T cells, B cells, natural killer cells, monocytes, and macrophages (3). Activated lymphocytes produce and release IL-2 receptor from the cell surface, which can be measured as soluble interleukin-2 receptor (sIL-2R). Multiple organ failure develops as a result of cytokine storm in some patients; circulating levels of sIL-2R are increased. Here, we report a case of thyroid storm with elevated levels of sIL-2R. The patient could not be rescued with plasma exchange for fulminant hepatitis. 


\section{Case presentation}

A 48-year-old man had fatigue for 3 months. One month before admission, he developed a low-grade fever, shortness of breath, and weight loss. He was admitted to the hospital because of tachycardia, liver dysfunction, hyperthyroidism, and cardiomegaly on chest x-ray. He had been prescribed antihypertensives for years. He had no history of heavy drinking, blood transfusion, or drug abuse. Blood tests revealed Graves' disease. Methimazole, inorganic iodide, hydrocortisone, and a $\beta$-blocker were started. On the day after admission, he became

Table 1 Summary of laboratory testing results.

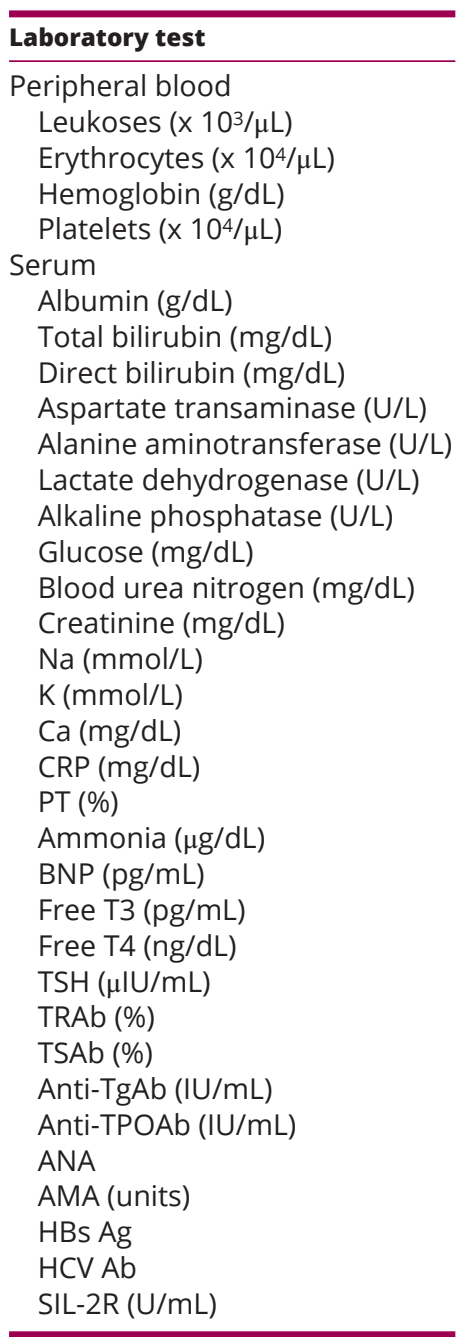

\begin{tabular}{|c|c|}
\hline Values & Reference range \\
\hline 11.1 & $3.3-8.6$ \\
\hline 434 & $435-555$ \\
\hline 13.1 & $13.7-16.8$ \\
\hline 28.9 & $15.8-34.8$ \\
\hline 2.56 & $4.1-5.1$ \\
\hline 2.5 & $0.4-1.5$ \\
\hline 1.2 & $0.1-0.3$ \\
\hline 1458 & $13-30$ \\
\hline 555 & $10-42$ \\
\hline 3266 & $124-222$ \\
\hline 370 & $103-322$ \\
\hline 35 & 73-109 \\
\hline 42.3 & $8-20$ \\
\hline 2.16 & $0.7-1.1$ \\
\hline 141 & $138-145$ \\
\hline 5.4 & $3.6-4.8$ \\
\hline 7.6 & $8.8-10.1$ \\
\hline 1.81 & $<0.14$ \\
\hline 19.1 & $80-100$ \\
\hline 203 & $12-66$ \\
\hline 397.3 & $<18.4$ \\
\hline 7.5 & $1.9-3.2$ \\
\hline 6.0 & $0.7-1.5$ \\
\hline$<0.01$ & $0.4-5.0$ \\
\hline 40 & $<15 \%$ \\
\hline 645 & $<120 \%$ \\
\hline 40.0 & 141106 \\
\hline $\begin{array}{c}29.9 \\
(-)\end{array}$ & $1.1-5.2$ \\
\hline $\begin{array}{c}<1.5 \\
(-) \\
(-)\end{array}$ & $<20$ \\
\hline 9770 & $145-519$ \\
\hline
\end{tabular}

AMA, anti-mitochondrial antibody; ANA, antinuclear antibody; Anti-TgAb., anti-thyroglobulin antibody; Anti-TPOAb, anti-thyroid peroxidase antibody; BNP, brain natriuretic peptide; CRP, C-reactive protein; $\mathrm{HBs} \mathrm{Ag}$, hepatitis B surface antigen; HCV Ab, anti-hepatitis C virus antibody; PT, prothrombin time; sIL-2R_soluble interleukin-2 receptor; T3, triiodothyronine; $T 4$, thyroxine; TRAb, thyroid-stimulating homione receptor antibody; TSAb, thyroid-stimulating antibody. unconscious with a high fever and was transferred to the intensive care unit after tracheal intubation.

\section{Investigation}

On examination, height was $164 \mathrm{~cm}$ and weight was $59 \mathrm{~kg}$. Temperature was $37.8^{\circ} \mathrm{C}$, blood pressure was $77 / 56 \mathrm{mmHg}$, and heart rate was 141 beats per minute. The Glasgow Coma Scale score was E1VTM1 (sedated, T indicates endotracheal intubation). He had diffuse goiter without ophthalmopathy, abnormal sweating, distended jugular veins, and bilateral pretibial pitting edema. Blood tests showed extremely high levels of aspartate transaminase and alanine aminotransferase (1458 and 555 U/L, respectively), renal failure, hypoglycemia, coagulopathy, and hyperammonemia (Table 1). Arterial blood analysis revealed hypoxia and metabolic acidosis. Chest radiography demonstrated cardiomegaly and decreased pulmonary permeability in the right lung field (Fig. 1A). Electrocardiography showed atrial flutter. Echocardiography revealed a visual ejection fraction of $20 \%$ with diffuse hypokinesis. Computed tomography (CT) demonstrated an enlarged thyroid gland (Fig. 1B), but there were no abnormal lesions in the liver (Fig. 1C) or the brain. The patient was diagnosed with thyroid storm based on criteria from the Japan Thyroid Association and Burch-Wartofsky Point Scale (100 points).

\section{Treatment}

Cardiac arrest occurred $3 \mathrm{~h}$ after admission to the intensive care unit. Chest compressions and initiation of intraaortic balloon pumping were performed for cardiogenic shock. Return of spontaneous circulation was achieved and cardioversion was performed for atrial flutter. Intravenous methimazole $(30 \mathrm{mg} /$ day), inorganic iodide $(100 \mathrm{mg} /$ day), hydrocortisone $(200 \mathrm{mg} /$ day), and landiolol hydrochloride $(7.2 \gamma)$ normalized thyroid function in 10 days (Fig. 2). Artificial respiratory management, intravenous glucose administration for hypoglycemia, and plasma exchange (including plasma filtration with dialysis and plasma adsorption) for hepatic failure resulted in the recovery of consciousness (E4V2M6). The patient was extubated on hospital day 10.

\section{Outcome and follow-up}

Transaminase levels decreased, but levels of total bilirubin increased and prothrombin time became prolonged (Fig. 2). The switch from methimazole to propylthiouracil was not effective. CT of the abdomen showed increased 

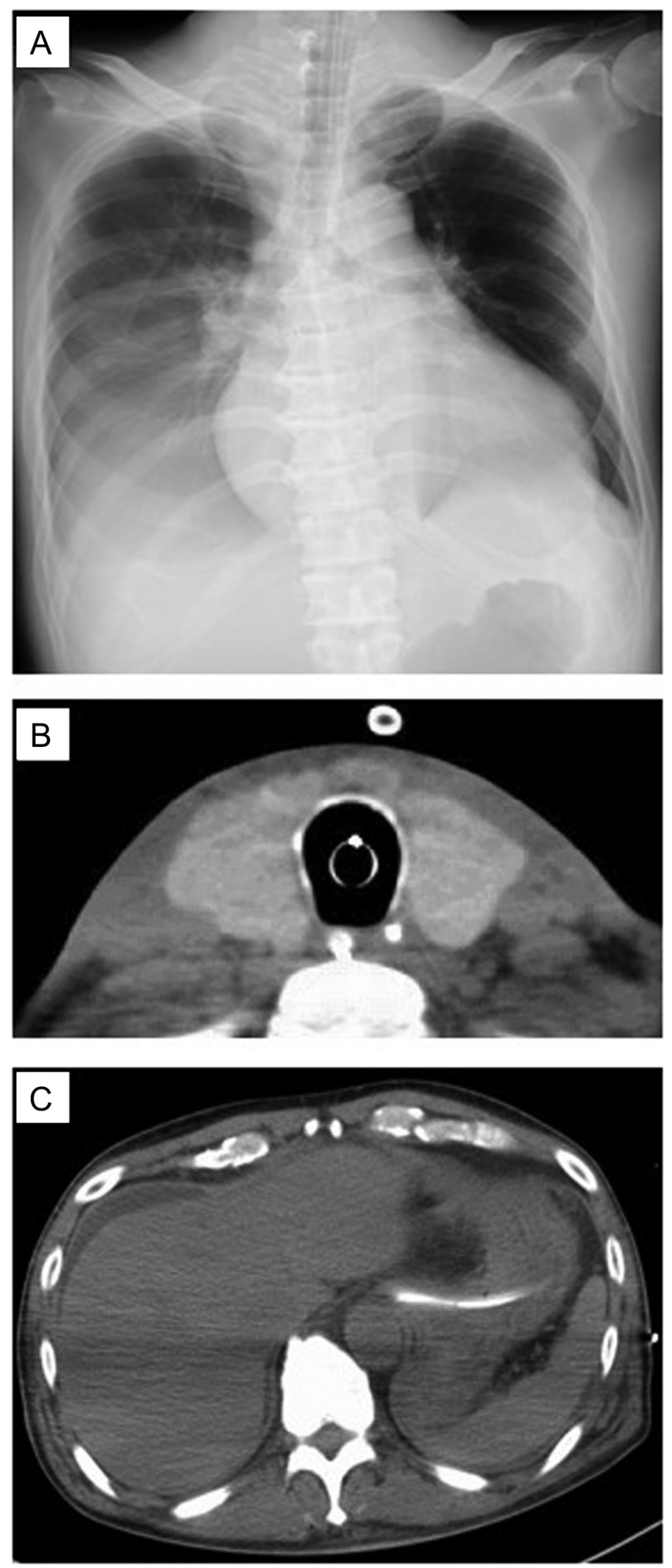

Figure 1

(A) Chest x-ray showed cardiomegaly and a pleural effusion. (B) Cervical computed tomography (CT) showed a swollen thyroid. (C) Abdominal CT showed ascites without any organic lesions in the liver.



\begin{tabular}{|l|c|c|c|c|c|}
\hline Laboratory Test & Day2 & Day10 & Day27 & Day39 & Day56 \\
\hline Free T3 (pg/mL) & 7.5 & 3.1 & 2.1 & 2.5 & 3.2 \\
\hline Free T4 (ng/dL) & 6.0 & 2.3 & 1.3 & 1.6 & 1.5 \\
\hline Total bilirubin (mg/dL) & 3.5 & 17.6 & 36.2 & 37.7 & 28.4 \\
\hline AST (U/L) & 11960 & 65 & 46 & 22 & 24 \\
\hline ALT (U/L) & 3087 & 67 & 49 & 14 & 2 \\
\hline PT (\%) & 8.9 & 37.3 & 28.6 & 26.6 & 20.7 \\
\hline Ammonia $(\mu \mathrm{g} / \mathrm{dL})$ & 112 & 47 & 55 & 86 & $>500$ \\
\hline
\end{tabular}

Abbreviations: ALT, alanine transaminase; AST, aspartate transaminase; GCS, Glasgow Coma Scale; PT, prothrombin time; T3, triiodothyronine; $\mathrm{T} 4$, thyroxine.

Figure 2

Clinical course.

ascites. Coagulopathy and jaundice worsened. The patient was diagnosed with fulminant hepatitis. Investigations showed no evidence of viral hepatitis, congestive heart failure, or adverse drug effects, except for elevated levels of sIL2-R. Imaging studies and skin biopsy to detect intravascular lymphoma revealed no evidence of malignancy, suggesting that elevated sIL2-R levels were induced by thyroid storm. Fulminant hepatitis was refractory to plasma exchange. CT of the abdomen demonstrated exacerbation of liver atrophy. There were no suitable donors for orthotopic liver transplantation. Gastrointestinal bleeding, intra-abdominal hemorrhage, hematuria, subcutaneous bleeding, and endotracheal bleeding were not controlled. Red blood cell, platelet, and plasma transfusions were not effective in treating hemoperitoneum and gastrointestinal bleeding. The patient died on hospital day 62 .

\section{Discussion}

We described a fatal case of thyroid storm complicated by fulminant hepatitis refractory to plasma exchange. 
Fulminant hepatitis induced by thyroid storm is rare but life threatening. A previous report of thyroid storm with fulminant hepatitis showed four of ten patients died with heart failure and elevated levels of bilirubin $(4,5,6)$. Orthotopic liver transplantation was performed in two of six patients who survived, but not in any patients who died $(4,5,6)$. In thyroid storm, heart failure causes liver dysfunction via hepatic hypoxia. A nationwide survey in Japan found that the most common causes of death in patients with thyroid storm were multiple organ failure and congestive heart failure (7). In our patient, cardiogenic shock caused multiple organ failure and was the most important factor leading to fulminant hepatitis and death.

Hypoglycemia is rarely associated with thyroid storm. Excess levels of thyroid hormone increase levels of counterregulatory hormones and decrease the ability of insulin to bind to its receptor. Hypoglycemia with thyroid storm is caused by malnutrition, adrenal failure, decreased insulin clearance due to renal failure, and insufficient gluconeogenesis due to hepatic failure (8). Our patient had not eaten for 3 days before admission and developed cardiac and hepatic failure due to hyperthyroidism, which was associated with severe hypoglycemia.

Plasma exchange has been reported to be effective for thyroid storm by reducing levels of thyroid hormones, TSH receptor antibodies, catecholamines, and cytokines (9). Severe hepatic or renal dysfunction is a poor prognostic marker, suggesting disease refractory to plasma exchange (9). In our case, plasma exchange, plasma filtration with dialysis, and plasma adsorption normalized thyroid hormone levels, but not liver function.

This patient had abnormally high circulating levels of sIL-2R. Some hematologic malignancies and autoimmune diseases are associated with elevated levels of sIL-2R (3). Plasma levels of thyroxine are correlated with levels of sIL-2R in patients with Graves' disease. Thyroxine directly stimulates T cell activation and proliferation (10). Elevated sIL-2R has been reported in patients with disseminated intravascular coagulation caused by thyroid storm (11). Our patient had high levels of sIL-2R and free thyroxine as well as multiple organ failure, suggesting that the immune response stimulated by thyroid storm resulted in high levels of sIL-2R. Circulating sIL-2R is not involved in the development of fulminant hepatitis, but the immuneinflammatory response from thyroid storm could induce multiple organ failure and fulminant hepatitis.

We describe a fatal case of thyroid storm with fulminant hepatitis and elevated levels of sIL-2R. Orthotopic liver transplantation is the preferred treatment for fulminant hepatitis due to thyroid storm (4). Excessively high circulating levels of sIL-2R might be a marker of poor prognosis in thyroid storm with fulminant hepatitis.

\section{Declaration of interest}

The authors declare that there is no conflict of interest that could be perceived as prejudicing the impartiality of the research reported.

\section{Funding}

This research did not receive any specific grant from any funding agency in the public, commercial, or not-for-profit sector.

\section{Patient consent}

Written informed consent was obtained from the patient's wife.

\section{Author contribution statement}

Y Tanaka, T Uchida, and $\mathrm{H}$ Yamaguchi wrote the case report. Y Kudo, $\mathrm{T}$ Yonekawa, and $\mathrm{M}$ Nakazato contributed to the clinical management of this patient. $\mathrm{K}$ Shimizu and $\mathrm{T}$ Ideguchi were involved in the management of this case.

\section{References}

1 Tietgens ST \& Leinung MC. Thyroid storm. Medical Clinics of North America 199579 169-184. (https://doi.org/10.1016/S00257125(16)30090-6)

2 Malic R \& Hodgson H. The relationship between the thyroid gland and the liver. QJM 200295 559-569. (https://doi.org/10.1093/ qjmed/95.9.559)

3 Rubin LA \& Nelson DL. The soluble inerleukin-2 receptor: biology, function, and clinical application. Annals of Internal Medicine 1990 113 619-627. (https://doi.org/10.7326/0003-4819-113-8-619)

4 Hambleton C, Buell J, Saggi B, Balart L, Shores NJ \& Kandil E. Thyroid storm complicated by fulminant hepatic failure: case report and literature review. Annals of Otology, Rhinology, and Laryngology 2013122 679-682. (https://doi.org/10.1177/000348941312201103)

5 Soleimanpour SA. Fulminant liver failure associated with delayed identification of thyroid storm due to heterophile antibodies. Clinical Diabetes and Endocrinology 20151 1-5. (https://doi.org/10.1186/ s40842-015-0012-6)

6 de Campos Mazo DF, de Vasconcelos GB, Pereira MA, de Mello ES, Bacchella T, Carrilho FJ \& Cançado EL. Clinical spectrum and therapeutic approach to hepatocellular injury in patients with hyperthyroidism. Clinical and Experimental Gastroenterology 20136 9-17. (https://doi.org/10.2147/CEG.S39358)

7 Akamizu T. Thyroid storm: a Japanese perspective. Thyroid 201828 32-40. (https://doi.org/10.1089/thy.2017.0243)

8 Homma M, Shimizu S, Ogata M, Yamada Y, Saito T \& Yamamoto T. Hypoglycemic coma masquerading thyrotoxic storm. Internal Medicine 199938 871-874. (https://doi.org/10.2169/ internalmedicine.38.871)

9 Muller C, Perrin P, Faller B, Richter S \& Chantrel F. Role of plasma exchange in the thyroid storm. Therapeutic Apheresis and Dialysis 201115 522-531. (https://doi.org/10.1111/j.17449987.2011.01003.x)

10 Jiskra J, Antosová M, Límanová Z, Telicka Z \& Lacinová Z. The relationship between thyroid function, serum monokine induced 
by interferon gamma and soluble interleukin-2 receptor in thyroid autoimmune diseases. Clinical and Experimental Immunology 2009 156 211-216. (https://doi.org/10.1111/j.1365-2249.2009.03897.x)

11 Shimoda Y, Satoh T, Takahashi H, Katano-Toki A, Ozawa A,

Tomaru T, Horiguchi N, Kaira K, Nishioka M, Shibusawa N et al. A case of thyroid storm with a markedly elevated level of circulating soluble interleukin-2 receptor complicated by multiple organ failure and disseminated intravascular coagulation syndrome. Endocrine Journal 201461 691-696. (https://doi.org/10.1507/endocrj.EJ140073)

Received in final form 21 August 2019

Accepted 6 September 2019 